\title{
Diversidade de Leguminosae em uma área de savana do estado de Roraima, Brasil
}

\author{
Andréia Silva Flores ${ }^{1}$ e Rodrigo Schütz Rodrigues ${ }^{2,3}$
}

Recebido em 16/09/2008. Aceito em 8/07/2009

RESUMO - (Diversidade de Leguminosae em uma área de savana estado de Roraima, Brasil). Leguminosae é considerada a família mais diversa nas savanas de Roraima, que são as maiores áreas de savana da Amazônia brasileira. Este trabalho tem como objetivos fornecer uma listagem florística e analisar os padrões de distribuição geográfica dos táxons de Leguminosae encontrados em uma área de savana em Boa Vista, Roraima $\left(02^{\circ} 52^{\prime} 07^{\prime \prime} \mathrm{N} ; 60^{\circ} 43^{\prime} 03^{\prime \prime} \mathrm{W}\right)$. Foram registrados 80 táxons distribuídos em 38 gêneros. Seis táxons são novos registros para a flora de Roraima. Os gêneros que apresentaram o maior número de espécies foram Aeschynomene e Chamaecrista, cada um com sete espécies. Leguminosae-Papilionoideae foi a subfamília mais diversa em número de gêneros (24) e de táxons infragenéricos (53). A análise da distribuição geográfica revelou a predominância de táxons com padrão neotropical. A área estudada representa um sítio diverso em táxons de Leguminosae, pois concentra cerca de $87 \%$ da diversidade citada para a família nas savanas de Roraima. Palavras-chave: fitogeografia, Guayana, levantamento florístico, Leguminosae

\begin{abstract}
Leguminosae diversity in a savanna area of Roraima, Brazil). Leguminosae is the most diverse family in the savannas of Roraima, which are the largest savanna areas in the Brazilian Amazon. This paper presents a floristic survey and an analysis of the geographic distribution patterns of Leguminosae taxa in a savanna area at Boa Vista, Roraima $\left(02^{\circ} 52^{\prime} 07^{\prime \prime} \mathrm{N} ; 60^{\circ} 43^{\prime} 03^{\prime \prime} \mathrm{W}\right)$. The survey identified 80 taxa belonging to 38 genera. Six taxa are new records for the flora of Roraima. The best-represented genera were Aeschynomene and Chamaecrista, both with seven species each. LeguminosaePapilionoideae was the most diverse subfamily based on the number of genera (24) and infrageneric taxa (53). The phytogeographical analysis revealed predominance of taxa with a Neotropical distribution. The savanna site analyzed represents a legume diverse area, because it concentrates $87 \%$ of legume diversity referred for the savannas of Roraima.
\end{abstract}

Key words: floristic survey, Guayana, Leguminosae, phytogeography

\section{Introdução}

Leguminosae é a terceira maior família de angiospermas, apresentando cerca de 18.000 espécies, distribuídas em aproximadamente 727 gêneros em três subfamílias: Caesalpinioideae, Mimosoideae e Papilionoideae (Lewis et al. 2005). Sua distribuição é cosmopolita e está bem representada nas regiões tropicais, subtropicais e temperadas.

Nas regiões fitogeográficas da Amazônia e da Guayana, inventários atestam a grande importância da família Leguminosae na composição florística de diferentes sítios (Salomão et al. 1988; Almeida et al. 1993; Terborgh \& Andresen 1998; Ferreira \& Prance 1998; Milliken 1998; Oliveira 2000; Miranda \& Absy 2000; Funk et al. 2007).

Roraima ocupa a região mais ao norte da Amazônia brasileira, fazendo fronteira internacional com a Guiana e a Venezuela. O Estado apresenta sua cobertura vegetacional original distribuída em diferentes formações florestais e nãoflorestais, incluindo formações vegetais particulares como as campinas e campinaranas concentradas ao sul e os tepuis, ao norte (Sette-Silva 1997; Barbosa et al. 2003).

Dentre os ecossistemas não-florestais, as savanas encontradas em Roraima se constituem como as maiores áreas de savanas da Amazônia brasileira, fazendo parte do complexo paisagístico "Rio Branco-Rupununi", que se estende para a Guiana e Venezuela (Jansen-Jacobs \& ter Steege 2000, Barbosa et al. 2007). Fitogeograficamente, estas savanas estão inseridas na região Guayana, sobre o Escudo das Guianas (Huber 2006). A flora fanerogâmica das savanas de Roraima, apesar das estimativas indicarem a presença de aproximadamente 500-600 espécies (Barbosa et al. 2007; Miranda \& Absy 1997), são geralmente consideradas de baixa riqueza específica, juntamente com os demais sítios disjuntos de savanas amazônicas (Miranda \& Absy 1997; Ratter et al. 2006).

Leguminosae é apontada como a família mais diversa nas savanas de Roraima (Miranda \& Absy 1997). Entretanto, as informações taxonômicas sobre a família ainda são fragmentadas e as estimativas de diversidade preliminares. Desta forma, este trabalho tem como objetivos fornecer a listagem florística e a análise dos padrões de distribuição geográfica dos táxons de Leguminosae encontrados em uma área de savana em Boa Vista, Roraima.

\section{Material e métodos}

Área de estudo - O trabalho foi realizado no Campus do Cauamé, pertencente ao Centro de Ciências Agrárias da Universidade Federal de Roraima, localizado no município de Boa Vista, Roraima $\left(02^{\circ} 52^{\prime} 07^{\prime \prime} \mathrm{N} \mathrm{e}\right.$ $\left.60^{\circ} 43^{\prime} 03^{\prime \prime} \mathrm{W}\right)$. Devido ao seu bom estado de conservação e por apresentar várias fitofisionomias de savana (Fig. 1), esta área foi incorporada ao Programa de Pesquisa em Biodiversidade - PPBIO Amazônia. Aárea apresenta cerca de 500 ha, compreendendo fitofisionomias de savana arbórea, parque, graminosa, bem como as matas ciliares do Rio Cauamé, afluente do Rio Branco. Tanto o ecossistema de savana quanto o de floresta, representado pelas matas ciliares, fazem parte da paisagem geral das savanas de Roraima (Barbosa \& Miranda 2005) e foram amostrados neste estudo.

De acordo com a classificação de Koeppen, o clima da região é o "Awi”, tropical chuvoso com 5-7 meses de estação seca. A precipitação média anual é de $1614 \mathrm{~mm}$ sendo a maior concentração verificada entre os meses de maio e junho e a menor, entre dezembro e março (Barbosa 1997). O relevo é suavemente ondulado e os solos são predominantemente pertencentes ao

\footnotetext{
1 Fundação Estadual do Meio Ambiente, Ciência e Tecnologia de Roraima, Museu Integrado de Roraima, Boa Vista, RR, Brasil

2 Universidade Federal de Roraima, Centro de Estudos da Biodiversidade, Boa Vista, RR, Brasil

3 Autor para correspondência: rodrigo@cbio.ufrr.br
} 

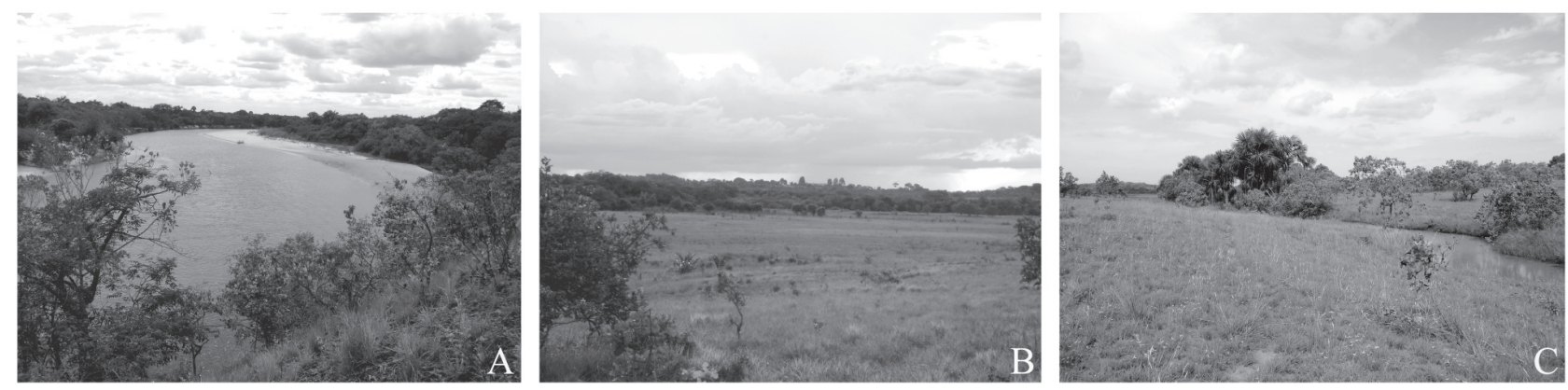

Figura 1. Vistas de algumas fitofisionomias encontradas na área de estudo (Boa Vista, Roraima, Brasil). A. Vista do rio Cauamé e de sua mata ciliar ao fundo. B Vista da savana graminosa, ao fundo mata ciliar do rio Cauamé. C. Vista de savana graminosa, ao fundo igarapé com buriti (Mauritia flexuosa L.f.) e savana parque com caimbé (Curatella americana L.). Fotos: Christiane Costa, 2008.

grupo dos latossolos em mosaico com os podzólicos (Radambrasil 1975).

Coleta e análise de dados - Foram realizadas coletas mensais durante os meses de setembro de 2006 a maio de 2008. As áreas foram percorridas de forma a cobrir a maior extensão possível das diferentes fitofisionomias em cada expedição. Os exemplares coletados foram incorporados aos herbários MIRR (Museu Integrado de Roraima) e UFRR (Universidade Federal de Roraima), ambos em Boa Vista. Além disso, foram analisados materiais dos herbários UFRR e MIRR provenientes da área de estudo.

As identificações das espécies e categorias infra-específicas foram baseadas em comparações com coleções-tipo e/ou fotografias das mesmas, obras originais ou revisões e descrições encontradas na literatura.

Os padrões de distribuição geográfica de cada táxon foram definidos com base em seus limites de distribuição (Queiroz 2006). Para tanto, informações sobre a distribuição de cada táxon foram extraídas principalmente de revisões taxonômicas, ou quando inexistentes, de floras regionais (Cowan \& Lindeman 1989; Barneby et al. 1998; 2001; Aymard et al. 1999). A partir destas informações foram estabelecidos os padrões de distribuição geográfica para os táxons nativos em quatro macrorregiões geográficas (adaptados de Kelloff \& Funk 2004), sendo mais detalhadas para os táxons ocorrentes na América do Sul. A delimitação da região fitogeográfica Guayana seguiu Funk et al. (2007). O termo "cerrado" empregado refere-se exclusivamente às áreas de savana do Brasil Central, excluindo-se as savanas Rio BrancoRupununi, de acordo com Pennington et al. (2006). Os dados referentes aos hábitos e as fitofisionomias onde os táxons ocorrem (savana, mata ciliar ou local alterado) foram obtidos das observações de campo e das informações contidas nas etiquetas das exsicatas analisadas.

\section{Resultados e discussão}

Foram encontrados 38 gêneros e 75 espécies de Leguminosae, das quais três apresentaram mais de um táxon infraespecífico, totalizando 80 táxons infragenéricos na área de estudo (Tab. 1). Papilionoideae foi a subfamília com maior número de representantes, tanto no número de gêneros (24), quanto de táxons infragenéricos (53). Na subfamília Caesalpiniodeae, foram encontrados oito gêneros e 17 táxons infragenéricos e em Mimosoideae, seis gêneros e dez táxons infragenéricos. Quatro espécies são exóticas em Roraima (Tab. 2) e seis táxons (indicados na Tab. 1 com asteriscos) são novas citações para a flora do Estado: Chamaecrista nictitans subsp. patellaria var. praetexta (Vogel) H.S. Irwin \& Barneby, Centrosema pascuorum Mart. ex Benth., Indigofera suffruticosa Mill., Rhynchosia melanocarpa Grear, Stylosanthes capitata Vogel e Vigna juruana (Harms) Verdc. Os gêneros que apresentaram o maior número de espécies foram Aeschynomene e Chamaecrista, ambos com sete espécies. Aproximadamente $60 \%$ dos gêneros apresentaram somente uma espécie, sendo estas principalmente arbóreas.

Nas diferentes fitofisionomias da área de estudo, as leguminosas ocorreram como ervas (21 táxons $-26,25 \%$ ), subarbustos ( 25 táxons $-31,25 \%)$, trepadeiras volúveis (6 táxons $-7,5 \%)$, arbustos ( 3 táxons $-3,75 \%$ ), árvores (21 táxons $-26,25 \%$ ), sendo que indivíduos de quatro espécies (5\%) podem ocorrer como ervas ou subarbustos.

As fitofisionomias de savana apresentaram a maior diversidade de leguminosas, com 56 táxons, onde foram encontradas todas as novas ocorrências para o estado, enquanto que nas matas ciliares foram registrados 19 táxons. De modo geral, no presente estudo, as espécies de leguminosas nãoarbóreas predominaram em áreas campestres das savanas, enquanto que as espécies arbóreas são frequentemente encontradas no interior ou borda das matas ciliares. Em áreas alteradas ocorreram todas as espécies exóticas e Cassia moschata Kunth, nativa na região Neotropical.

A análise da distribuição geográfica dos 76 táxons nativos revelou a ocorrência de quatro padrões amplos de distribuição: 1) Pantropical (PAN) com dois táxons (2,63\%); 2) Anfiatlântico (AfA), com três táxons (3,95\%); 3) Neotropical (NEO), com 46 táxons $(60,53 \%)$ e 4) América do Sul (AmS), com 25 táxons (32,89\%). Para os táxons que ocorrem somente na América do Sul, ainda podem ser reconhecidos os seguintes padrões: 4.1) distribuição ampla na América do Sul (AmS Amplo), com nove táxons (11,85\%); 4.2) distribuição coincidente com a bacia Amazônica, com extensões para a região fitogeográfica Guayana (AmS Norte), com oito táxons (10,52\%) e 4.3) distribuição restrita ou predominante na região fitogeográfica Guayana (AmS Guayana), com oito táxons (10,52\%) (Tab. 2).

A grande predominância de espécies de ampla distribuição na América distingue a área de estudo de alguns sítios de cerrado e caatinga estudados sob o aspecto fitogeográfico. Filardi et al. (2007) constataram que, dentre os 41 táxons de Leguminosae-Papilionoideae da Serra da Canastra, Minas Gerais, somente $14,6 \%$ apresentam o padrão neotropical, enquanto que para a área de estudo, tomando-se somente os táxons desta subfamília, este valor chega a 54,7\%. Similarmente, Queiroz (2006), ao determinar o padrão de distribuição geográfica de 274 táxons de Leguminosae da 
Tabela 1. Listagem dos táxons de Leguminosae de uma área de savana em Boa Vista, Roraima, Brasil. Novas ocorrências para a flora de Roraima são indicadas por asteriscos.

\begin{tabular}{|c|c|}
\hline Táxon & Material testemunho \\
\hline \multicolumn{2}{|l|}{ LEGUMINOSAE-CAESALPINIOIDEAE } \\
\hline Bauhinia ungulata $\mathrm{L}$. & Flores et al. 1373 (MIRR) \\
\hline Cassia moschata Kunth & Rodrigues 1617 (MIRR) \\
\hline Chamaecrista desvauxii var. brevipes (Benth.) H.S. Irwin \& Barneby & Flores et al. 1601 (MIRR) \\
\hline C. diphylla (L.) Greene & Flores et al. 1693 (MIRR) \\
\hline C. flexuosa (L.) Greene & Flores et al. 1353 (MIRR) \\
\hline C. hispidula (Vahl) H.S. Irwin \& Barneby & Flores et al. 1700 (MIRR) \\
\hline C. nictitans ssp. disadena (Steud.) H.S. Irwin \& Barneby var. disadena & Rodrigues et al. 1785 (MIRR) \\
\hline C. nictitans ssp. patellaria (Collad.) H.S. Irwin \& Barneby var. patellaria & Rodrigues et al. 1784 (MIRR) \\
\hline C. nictitans ssp. patellaria var. praetexta (Vogel) H.S. Irwin \& Barneby * & Flores et al. 1387 (MIRR) \\
\hline C. rotundifolia (Pers.) Greene var. rotundifolia & Flores et al. 1348 (MIRR) \\
\hline C. serpens (L.) Greene var. serpens & Rodrigues et. al 1769 (MIRR) \\
\hline Copaifera pubiflora Benth. & Flores et al. 1374 (MIRR) \\
\hline Macrolobium acaciifolium (Benth.) Benth. & Flores et al. 1378 (MIRR) \\
\hline M. multijugum (DC.) Benth. var. multijugum & Flores et al. 1405 (MIRR) \\
\hline Martiodendron excelsum (Benth.) Gleason & Rodrigues et al. 1618 (MIRR) \\
\hline Peltogyne paniculata ssp. pubescens (Benth.) M.F. Silva & Flores et al. 1623 (MIRR) \\
\hline Senna obtusifolia (L.) H.S. Irwin \& Barneby & Rodrigues et al. 1791 (MIRR) \\
\hline \multicolumn{2}{|l|}{ LEGUMINOSAE-MIMOSOIDEAE } \\
\hline Hydrochorea corymbosa (Rich.) Barneby \& J.W. Grimes & Flores et al. 1391 (MIRR) \\
\hline Inga cf. pilosula (Rich.) J.F. Macbr. & Flores et al. 1417 (MIRR) \\
\hline Leucaena leucocephala (Lam.) de Wit & Flores et al. 1349 (MIRR) \\
\hline Mimosa caesalpiniifolia Benth. & Rodrigues et al. 1811 (MIRR) \\
\hline M. camporum Benth. & Flores et al. 1473 (MIRR) \\
\hline M. debilis Humb. \& Bonpl. ex Willd. var. debilis & Flores et al. 1342 (MIRR) \\
\hline M. pudica var. tetrandra (Humb. \& Bonpl. ex Willd.) DC. & Rodrigues et al. 1775 (MIRR) \\
\hline M. quadrivalvis var. leptocarpa (DC.) Barneby & Flores et al. 1697 (MIRR) \\
\hline Neptunia plena (L.) Benth. & Flores et al. 1703 (MIRR) \\
\hline Zygia cataractae (Kunth) L. Rico & Flores et al. 1482 (MIRR) \\
\hline \multicolumn{2}{|l|}{ LEGUMINOSAE-PAPILIONOIDEAE } \\
\hline Aeschynomene americana L. var. americana & Flores et al. 1696 (MIRR) \\
\hline A. brasiliana (Poir.) DC. var. brasiliana & Rodrigues et al. 1762 (MIRR) \\
\hline A. filosa Mart. ex Benth. & Flores et al. 1712 (MIRR) \\
\hline A. histrix var. densiflora (Benth.) Rudd & Flores et al. 1624 (MIRR) \\
\hline A. histrix Poir. var. histrix & Rodrigues et al. 1778 (MIRR) \\
\hline A. histrix var. incana (Vogel) Benth. & Rodrigues et al. 1780 (MIRR) \\
\hline A. interrupta Benth. & Flores et al. 1509 (MIRR) \\
\hline A. paniculata Willd. ex Vogel & Flores et al. 1590 (MIRR) \\
\hline A. pratensis var. caribea Rudd & Flores et al. 1687 (MIRR) \\
\hline Bowdichia virgilioides Kunth & Flores et al. 1680 (MIRR) \\
\hline
\end{tabular}


Tabela 1. Continuação

\begin{tabular}{|c|c|}
\hline Táxon & Material testemunho \\
\hline Calopogonium mucunoides Desv. & Flores et al. 1346 (MIRR) \\
\hline Centrosema macrocarpum Benth. & Rodrigues et al. 1796 (MIRR) \\
\hline C. pascuorum Mart. ex Benth. * & Rodrigues et al. 1766 (MIRR) \\
\hline Clitoria fairchildiana Howard & Rodrigues et al. 1793 (MIRR) \\
\hline C. falcata Lam. & Flores et al. 1505 (MIRR) \\
\hline C. guianensis (Aubl.) Benth. & Flores et al. 1485 (MIRR) \\
\hline C. laurifolia Poir. & Rodrigues et al. 1781 (MIRR) \\
\hline Crotalaria maypurensis Kunth & Flores et al. 1603 (MIRR) \\
\hline C. pilosa Mill. & Flores et al. 1359 (MIRR) \\
\hline C. stipularia Desv. & Flores et al. 1619 (MIRR) \\
\hline Dalbergia inundata Spruce ex Benth. & Flores et al. 1404 (MIRR) \\
\hline D. revoluta Ducke & Flores et al. 1628 (MIRR) \\
\hline Desmodium barbatum (L.) Benth. & Rodrigues et al. 1800 (MIRR) \\
\hline D. distortum (Aubl.) J.F. Macbr. & Rodrigues et al. 1798 (MIRR) \\
\hline D. glabrum (Mill.) DC. & Rodrigues et al. 1801 (MIRR) \\
\hline D. procumbens (Mill.) A. Hitchc. & Rodrigues et al. 1799 (MIRR) \\
\hline D. triflorum (L.) DC. & Flores et al. 1891 (MIRR) \\
\hline Eriosema crinitum (Kunth) G. Don var. crinitum & Flores et al. 1589 (MIRR) \\
\hline Eriosema crinitum var. stipulare (Benth.) Fortunato & Rodrigues et al. 1763 (MIRR) \\
\hline E. simplicifolium (Kunth) G. Don var. simplicifolium & Flores et al. 1682 (MIRR) \\
\hline Galactia jussiaeana Kunth var. jussiaeana & Flores et al. 1455 (MIRR) \\
\hline Hymenolobium petraeum Ducke & Pessoni et al. 684 (UFRR) \\
\hline Indigofera hirsuta $\mathrm{L}$. & Rodrigues et al. 1788 (MIRR) \\
\hline I. lespedezioides Kunth & Flores et al. 1357 (MIRR) \\
\hline I. suffruticosa Mill. * & Rodrigues et al. 1805 (MIRR) \\
\hline Leptolobium nitens Vogel & Rodrigues et al. 1616 (MIRR) \\
\hline Machaerium inundatum (Mart. ex Benth.) Ducke & Flores et al. 1385 (MIRR) \\
\hline Macroptilium gracile (Poepp. ex Benth.) Urb. & Flores et al. 1591 (MIRR) \\
\hline Ormosia smithii Rudd & Flores et al. 1637 (MIRR) \\
\hline Pterocarpus rohrii Vahl & Pessoni et al. 615 (UFRR) \\
\hline Rhynchosia melanocarpa Grear * & Rodrigues et al. 1797 (MIRR) \\
\hline Soemmeringia semperflores Mart. & Flores et al. 1705 (MIRR) \\
\hline Stylosanthes angustifolia Vogel & Rodrigues et al. 1813 (MIRR) \\
\hline S. capitata Vogel * & Flores et al. 1371 (MIRR) \\
\hline S. guianensis var. gracilis (Kunth) Vogel & Flores et al. 1599 (MIRR) \\
\hline S. humilis Kunth & Flores et al. 1347 (MIRR) \\
\hline Swartzia dipetala Willd. ex Vogel & Flores et al. 1402 (MIRR) \\
\hline Swartzia latifolia var. sylvestris Cowan & Rodrigues et al. 1814 (MIRR) \\
\hline Tephrosia adunca Benth. & Flores et al. 1521 (MIRR) \\
\hline Vigna juruana (Harms) Verdc. * & Flores et al. 1533 (MIRR) \\
\hline V. longifolia (Benth.) Verdc. & Flores et al. 1698 (MIRR) \\
\hline Zornia crinita (Mohlenbr.) Vanni & Rodrigues et al. 1783 (MIRR) \\
\hline Z. latifolia Sm. & Rodrigues et al. 1806 (MIRR) \\
\hline
\end{tabular}


Tabela 2. Hábito, habitat e padrão de distribuição geográfica dos táxons de Leguminosae de uma área de savana em Roraima, Brasil. Hábito: erva (ERV), subarbusto (SUB), arbusto (ARB), árvore (ARV), trepadeira (TRE). Habitat: savana (SAV), mata ciliar (MTC), local alterado (ALT). Padrão de distribuição geográfica: Exótico - África/Ásia (EXO), Pantropical (PAN), Anfiatlântico (AfA), Neotropical (NEO), América do Sul (AmS). Para os táxons exclusivos da América do Sul são referidos os com distribuição: ampla na América do Sul (AmS Amplo), coincidente com a bacia Amazônica, com extensões para a região Guayana (AmS Norte) e restrita ou predominante na região Guayana (AmS Guayana).

\begin{tabular}{|c|c|c|c|}
\hline Táxon & Hábito & Habitat & Padrão distribuição \\
\hline \multicolumn{4}{|l|}{ LEGUMINOSAE-CAESALPINIOIDEAE } \\
\hline Bauhinia ungulata & $\mathrm{ARB}$ & MTC & NEO \\
\hline Cassia moschata & ARV & ALT & NEO \\
\hline Chamaecrista desvauxii var. brevipes & SUB & SAV & NEO \\
\hline C. diphylla & ERV & SAV & NEO \\
\hline C.flexuosa & SUB & SAV & NEO \\
\hline C. hispidula & ERV & SAV & NEO \\
\hline C. nictitans ssp. disadena var. disadena & SUB & SAV & NEO \\
\hline C. nictitans ssp. patellaria var. patellaria & SUB & SAV & NEO \\
\hline C. nictitans ssp. patellaria var. praetexta & SUB & SAV & NEO \\
\hline C. rotundifolia var. rotundifolia & ERV & SAV & NEO \\
\hline C. serpens var. serpens & ERV & SAV & NEO \\
\hline Copaifera pubiflora & ARV & MTC & AmS (Guayana) \\
\hline Macrolobium acaciifolium & ARV & MTC & AmS (Norte) \\
\hline M. multijugum var. multijugum & ARV & MTC & AmS (Norte) \\
\hline Martiodendron excelsum & ARV & MTC & AmS (Guayana) \\
\hline Peltogyne paniculata ssp. pubescens & ARV & MTC & AmS (Guayana) \\
\hline Senna obtusifolia & SUB & SAV & NEO \\
\hline \multicolumn{4}{|l|}{ LEGUMINOSAE-MIMOSOIDEAE } \\
\hline Hydrochorea corymbosa & ARV & MTC & AmS (Norte) \\
\hline Inga cf. pilosula & ARV & MTC & AmS (Norte) \\
\hline Leucaena leucocephala & ARV & ALT & $\mathrm{EXO}$ \\
\hline Mimosa caesalpiniifolia & $\mathrm{ARB}$ & ALT & $\mathrm{EXO}$ \\
\hline M. camporum & SUB & SAV & NEO \\
\hline M. debilis var. debilis & SUB & SAV & NEO \\
\hline M. pudica var. tetrandra & SUB & SAV & NEO \\
\hline M. quadrivalvis var. leptocarpa & SUB & SAV & NEO \\
\hline Neptunia plena & ERV & SAV & NEO \\
\hline Zygia cataractae & ARV & MTC & AmS (amplo) \\
\hline \multicolumn{4}{|l|}{ LEGUMINOSAE-PAPILIONOIDEAE } \\
\hline Aeschynomene americana var. americana & SUB & SAV & NEO \\
\hline A. brasiliana var. brasiliana & ERV & SAV & NEO \\
\hline A. filosa & SUB & SAV & NEO \\
\hline A. histrix var. densiflora & ERV & SAV & NEO \\
\hline A. histrix var. histrix & ERV & SAV & NEO \\
\hline A. histrix var. incana & ERV & SAV & NEO \\
\hline A. interrupta & $\mathrm{ARB}$ & MTC & AmS (Guayana) \\
\hline A. paniculata & SUB & SAV & NEO \\
\hline A. pratensis var. caribea & ERV/SUB & SAV & NEO \\
\hline
\end{tabular}


Tabela 2. Continuação

\begin{tabular}{|c|c|c|c|}
\hline Táxon & Hábito & Habitat & Padrão distribuição \\
\hline Bowdichia virgilioides & ARV & SAV & AmS (Amplo) \\
\hline Calopogonium mucunoides & TRE & SAV & NEO \\
\hline Centrosema macrocarpum & TRE & SAV & NEO \\
\hline C. pascuorum & ERV & SAV & NEO \\
\hline Clitoria fairchildiana & ARV & ALT & EXO \\
\hline C. falcata & TRE & SAV & AfA \\
\hline C. guianensis & SUB & SAV & NEO \\
\hline C. laurifolia & SUB & SAV & AmS (Amplo) \\
\hline Crotalaria maypurensis & SUB & SAV & NEO \\
\hline C. pilosa & SUB & SAV & NEO \\
\hline C. stipularia & ERV/SUB & SAV & NEO \\
\hline Dalbergia inundata & $\mathrm{ARV}$ & MTC & AmS (Norte) \\
\hline D. revoluta & ARV & MTC & AmS (Guayana) \\
\hline Desmodium barbatum & ERV & SAV & NEO \\
\hline D. distortum & SUB & SAV & PAN \\
\hline D. glabrum & SUB & SAV & NEO \\
\hline D. procumbens & ERV & SAV & PAN \\
\hline D. triflorum & ERV & ALT & EXO \\
\hline Eriosema crinitum var. crinitum & SUB & SAV & NEO \\
\hline Eriosema crinitum var. stipulare & SUB & SAV & NEO \\
\hline E. simplicifolium var. simplicifolium & ERV/SUB & SAV & NEO \\
\hline Galactia jussiaeana var. jussiaeana & SUB & SAV & AmS (Amplo) \\
\hline Hymenolobium petraeum & ARV & MTC & AmS (Norte) \\
\hline Indigofera hirsuta & SUB & SAV & NEO \\
\hline I. lespedezioides & SUB & SAV & NEO \\
\hline I. suffruticosa & SUB & SAV & NEO \\
\hline Leptolobium nitens & ARV & MTC & AmS (Norte) \\
\hline Machaerium inundatum & ARV & MTC & AmS (Norte) \\
\hline Macroptilium gracile & ERV & SAV & NEO \\
\hline Ormosia smithii & ARV & MTC & AmS (Guayana) \\
\hline Pterocarpus rohrii & ARV & MTC & NEO \\
\hline Rhynchosia melanocarpa & TRE & SAV & AmS (Amplo) \\
\hline Soemmeringia semperflores & ERV & SAV & AmS (Amplo) \\
\hline Stylosanthes angustifolia & ERV & SAV & AmS (Amplo) \\
\hline S. capitata & ERV & SAV & AmS (Amplo) \\
\hline S. guianensis var. gracilis & ERV & SAV & NEO \\
\hline S. humilis & ERV & SAV & NEO \\
\hline Swartzia dipetala & ARV & MTC & AmS (Guayana) \\
\hline Swartzia latifolia var. sylvestris & ARV & MTC & AmS (Guayana) \\
\hline Tephrosia adunca & ERV/SUB & SAV & AmS (Amplo) \\
\hline Vigna juruana & TRE & SAV & AfA \\
\hline$V$. longifolia & TRE & SAV & NEO \\
\hline Zornia crinita & ERV & SAV & NEO \\
\hline Z. latifolia & ERV & SAV & AfA \\
\hline
\end{tabular}


Caatinga, apontou que apenas 14,23\% dos táxons apresentam distribuição neotropical.

Os táxons registrados neste estudo que são restritos à região fitogeográfica Guayana ocorreram somente na mata ciliar e são árvores, duas Leguminosae-Caesalpinoideae [Martiodendron excelsum (Benth.) Gleason e Peltogyne paniculata subsp. pubescens (Benth.) M.F. Silva] e quatro Leguminosae-Papilionoideae [Dalbergia revoluta Ducke, Ormosia smithii Rudd, Swartzia dipetala Willd. ex Vogel e S. latifolia var. sylvestris Cowan]. A exceção foi Aeschynomene interrupta Benth., espécie arbustiva com flores lilases, que ocorre somente nas savanas Rio Branco-Rupununi (Brasil-Roraima e Guiana) (Fernandes 1996; Funk et al. 2007). Copaifera pubiflora Benth. é uma espécie arbórea amplamente encontrada na região Guayana, apresentando poucas extensões na Venezuela e Colômbia (Martins-daSilva et al. 2008). Nenhum táxon endêmico de Roraima foi encontrado na área de estudo. Dalbergia guttembergii A.M. de Carvalho, restrita à região de savana de Roraima, foi citada para a região do Rio Cauamé por Carvalho (1997), porém não foi encontrada no presente estudo. Esta espécie é um arbusto escandente ou arvoreta, diferindo das duas espécies de Dalbegia encontradas na área pela presença de flores amarelas ou brancas.

$\mathrm{Na}$ área de estudo, comparando-se as informações de hábito com as de habitat e os padrões de distribuição geográfica dos táxons, observa-se que todos os 55 táxons herbáceos e subarbustivos nativos de Leguminosae ocorreram nas fitofisionomias de savana. Para estes táxons, o padrão de distribuição neotropical foi fortemente predominante: 100\% nas subfamílias Caesalpinioideae e Mimosoideae e $73,5 \%$ em Papilionoideae. Todos os táxons herbáceos com hábito volúvel (trepadeiras) pertencem à Papilionoideae e ocorreram na fitofisionomia de savana, apresentando o padrão de distribuição neotropical como o predominante (50\%). Com relação aos táxons arbustivo-arbóreos de Leguminosae, observa-se que todos ocorreram na fitofisionomia de mata ciliar, exceto Bowdichia virgilioides Kunth, que ocorre nas fitofisionomias de savana. Esta espécie é amplamente encontrada no cerrado brasileiro em diferentes fitofisionomias como matas ciliares, cerradão, cerrado stricto sensu, campo rupestre, entre outras (Ratter et al. 2006; Mendonça et al. 2008), bem como em florestas estacionais secas do leste da América do Sul (Oliveira-Filho et al. 2006). Para os 21 táxons arbustivoarbóreos nativos, os padrões de distribuição geográfica predominantes foram AmS (Norte) e AmS (Guayana), ambos com oito táxons cada $(38,1 \%$ cada), enquanto que os três táxons com distribuição neotropical representam somente $14,3 \%$.

Uma análise separando-se os táxons nativos pelo tipo de habitat revelou diferenças nos padrões de distribuição geográfica entre os que ocorrem na mata ciliar e os que habitam a savana. Observa-se que os táxons ocorrentes na mata ciliar apresentam distribuição predominantemente restrita ao norte da América do Sul, agrupando-se os padrões AmS Norte e Guayana (ca. 79\%), diferentemente dos táxons encontrados na savana, que marcadamente têm um padrão de distribuição neotropical (ca. 77\%).

Para as savanas de Roraima, foram levantados 92 táxons de Leguminosae (Miranda \& Absy 1997; 2000), 29 pertencentes à subfamília Caesalpinioideae, nove à Mimosoideae e 56 à Papilionoideae. Nas duas últimas subfamílias, o número de táxons encontrados na área de estudo corresponde aproximadamente ao registrado para as savanas roraimenses, enquanto que em Caesalpinioideae este número é menor. Isto pode ser principalmente devido ao fato de que vários nomes citados nestas listagens são sinônimos, principalmente os citados sob Cassia s.l. Ainda para Roraima, Lewis \& Owen (1989) encontraram 121 táxons no levantamento de Leguminosae na Ilha de Maracá. Desta forma, o número de táxons encontrados na área de estudo corresponde à cerca de $66 \%$ do número de táxons da Ilha de Maracá, que concentra diferentes formações florestais e savânicas nos seus 92.000 ha de extensão.

Uma comparação do número de táxons de LeguminosaePapilionoideae encontrados na área de estudo com o de algumas áreas de savana e de florestas estacionais secas do Brasil já inventariadas, indica que, na área do Cauamé, há uma grande concentração de táxons em uma área comparativamente pequena, compatível ou mesmo superando outras regiões reconhecidamente ricas em táxons de Papilionoideae (Zappi et al. 2003; Queiroz 2004; Dutra et al. 2005; Silva 2005; Cardoso \& Queiroz 2007; Filardi et al. 2007). Observa-se também que os principais gêneros de Leguminosae em número de espécies na área do Cauamé (Chamaecrista e Aeschynomene) são predominantemente campestres e também muito diversos nas áreas de cerrado e de caatinga, onde também marcadamente se destacam Mimosa e Senna (Mendonça et al. 1998; 2008; Queiroz 2006). Entretanto, gêneros como Arachis, Caesalpinia, Calliandra e Senegalia não foram encontrados na área estudada e ocorrem amplamente na caatinga e cerrado, onde apresentam um grande número de espécies (Mendonça et al. 1998; 2008; Queiroz 2006), ao contrário do que é encontrado para as savanas de Roraima (Miranda \& Absy 1997; 2000).

Em conclusão, a área estudada representa um sítio diverso em táxons de Leguminosae, pois concentra cerca de $87 \%$ da diversidade da família citada para as savanas de Roraima. Além disso, a riqueza específica de Papilionoideae está entre as maiores referidas para áreas de savana do Brasil. Este elevado número de espécies de Leguminosae na região do Cauamé ressalta a necessidade de se intensificar coletas em outras regiões de savana do Estado, uma vez que esta vegetação ainda é muito pouco estudada floristicamente e considerada de baixa riqueza específica.

\section{Agradecimentos}

Os autores agradecem à Fundação Estadual do Meio Ambiente, Ciência e Tecnologia de Roraima pela concessão de uma bolsa DCR (FEMACT/ 
$\mathrm{CNPq}$ ) à primeira autora e pelo apoio institucional nas coletas de material; Christiane Costa (MIRR), Karuliny Maia (UFRR), Octaviano Grigio Jr. (MIRR), Roberto Serafim (MIRR) pelo valioso auxílio nas excursões de coleta e preparação do material; Christiane Costa pela gentileza em nos permitir publicar suas fotografias; aos dois assessores anônimos pela revisão do manuscrito. Este trabalho foi financiado pela CAPES/UFRR através do Programa de Auxílio à Pesquisa dos Recém Pós-Graduados - PROPESQUISA (Edital nº 19/2007- PRPPG).

\section{Referências bibliográficas}

Almeida, S.S.; Lisboa, P.L.B. \& Silva, A.S.L. 1993. Diversidade florística de uma comunidade arbórea na Estação científica "Ferreira Penna", em Caxiuanã (Pará). Boletim do Museu Paraense Emílio Goeldi, série Botânica 9: 93-128.

Aymard, G.A.; Cuello, N.L.; Berry, P.E.; Rudd, V.E.; Cowan, R.S.; Fantz, P.R.; Maxwell, R.H.; Stirton, C.H.; Poppendieck, H.-H.; Lima, H.C.; Fortunato, R.H.; Stergios, B.; Enrich, N.X.; Neill, D.A.; Pennington, R.T. \& Gil, C. 1999. Fabaceae. Pp. 231-433. In: Berry, P.E.; Yatskievych, K. \& Holst, B.K. (eds.). Flora of the Venezuelan Guayana. v. 5 Eriocaulaceae-Lentibulariaceae. St. Louis, Missouri Botanical Garden Press.

Barbosa, R.I. 1997. Distribuição das chuvas em Roraima. Pp. 325-335. In: R.I. Barbosa; E.J.G. Ferreira \& E.G. Castellõn (eds.). Homem, ambiente e ecologia no estado de Roraima. Manaus, INPA.

Barbosa, R.I.; Campos, C.; Pinto, F. \& Fearnside, P.M. 2007. The "Lavrados" of Roraima: Biodiversity and Conservation of Brazil's Amazonian Savannas. Functional Ecosystems and Communities 1: $29-41$.

Barbosa, R.I. \& Miranda, I.S. 2005. Fitofisionomias e diversidade vegetal nas savanas de Roraima. Pp. 61-78. In: R.I Barbosa; H.A.M. Xaud \& J.M. Costa e Souza (eds.) Savanas de Roraima: etnoecologia, biodiversidade e potencialidades agrossilvipastoris. Boa Vista, Femact.

Barbosa, R.I.; Xaud, M.R.; Silva, G.N. \& Cattâneo, A.C. 2003. Forest fires in Roraima, Brazilian Amazonia, International Forest Fire News 28: 51-66.

Barneby, R.C.; Stergios, B.; Cowan, R.S.; Berry, P.E.; Zarucchi, J.L.; Wunderlin, R.P.; Kearns, D.M.; Silva, M.F.; Tavares, A.S.; Velásquez, D.; Xena, N. \& Aymard, G.A. 1998. Caesalpiniaceae. Pp. 1-121. In: Berry, P.E.; Holst, B.K. \& Yatskievych, K. (eds.). Flora of the Venezuelan Guayana. v. 4 Caesalpiniaceae-Ericaceae. St. Louis, Missouri Botanical Garden Press.

Barneby, R.C.; Grimes, J.W.; Berry, P.E.; Brunner, D.; Forero, E.; Cárdenas, L.; Martino, G.; Hopkins, H.C.F. \& Occhioni, E.M.L. 2001. Mimosaceae. Pp. 580-686. In: Berry, P.E.; Yatskievych, K. \& Holst, B.K. (eds.). Flora of the Venezuelan Guayana. v. 6 Liliaceae-Myrsinaceae. St. Louis, Missouri Botanical Garden Press.

Cardoso, D.B.O.S. \& Queiroz, L.P. 2007. Diversidade de Leguminosae nas caatingas de Tucano, Bahia: implicações para a fitogeografia do SemiÁrido do Nordeste do Brasil. Rodriguésia 58: 379-391.

Carvalho, A.M. 1997. A synopsis of the genus Dalbergia (Fabaceae: Dalbergieae) in Brazil. Brittonia 49: 87-109.

Cowan, R.S. \& Lindeman, J.C. 1989. Caesalpiniaceae p.p. In: Görts-Van Rijn, A.R.A. (ed.). Flora of the Guianas. Series A: Phanerogams, Fascicle 7. Koenigstein: Koeltz Scientific Books.

Dutra, V.F.; Messias, M.C.T.B. \& Garcia, F.C.P. 2005 Papilionoideae (Leguminosae) nos campos ferruginosos do Parque Estadual do Itacolomi, Minas Gerais, Brasil: florística e fenologia. Revista Brasileira de Botânica 28: 493-504.

Fernandes, A. 1996. O táxon Aeschynomene no Brasil. Fortaleza, EUFC.

Ferreira, L.V. \& Prance, G.T. 1998. Structure and species richness of lowdiversity floodplain forest on the Rio Tapajós, Eastern Amazonia, Brazil. Biodiversity and Conservation 7: 585-596.

Filardi, F.L.R.; Garcia, F.C.P.; Dutra, V.F. \& São-Thiago, P.S. 2007. Papilionoideae (Leguminosae) do Parque Nacional da Serra da Canastra, Minas Gerais, Brasil. Hoehnea 34: 383-408.

Funk, V.; Hollowell, T.; Berry, P.; Kelloff, C. \& Alexander, N.S. 2007. Checklist of the plants of the Guiana Shield (Venezuela: Amazonas,
Bolivar, Delta Amacuro; Guyana, Surinam, French Guiana). Contributions from the United States National Herbarium 55: $1-584$.

Huber, O. 2006. Herbaceous ecosystems on the Guayana Shield, a regional overview. Journal of Biogeography 33: 464-475.

Jansen-Jacobs, M. \& ter Steege, H. 2000. Southwest Guyana: a complex mosaic of savannahs and forests. Pp.147-157. In: H. ter Steege (ed.). Plant Diversity in Guyana. Wageningen, Tropenbos Foundation.

Kelloff, C.L. \& Funk, V.A. 2004. Phytogeography of the Kaieteur Falls, Potaro Plateau, Guyana: floral distributions and affinities. Journal of Biogeography 31: 501-513.

Lewis, G.P. \& Owen, P.E. 1989. Legumes of the Iltha de Maracá. Kew, Royal Botanic Gardens.

Lewis, G.; Schrire, B.; Mackinder, B. \& Lock, M. (eds.). 2005. Legumes of the World. Kew, Royal Botanic Gardens.

Martins-da-Silva, R.C.V.; Pereira, J.F. \& Lima, H.C. 2008. O gênero Copaifera (Leguminosae - Caesalpinioideae) na Amazônia Brasileira. Rodriguésia 59: 455-476.

Mendonça, R.C.; Felfili, J.M.; Walter, B.M.T.; Silva Júnior, M.C.; Rezende, A.V.; Filgueiras, T.S. \& Nogueira, P.E. 1998. Flora vascular do cerrado. Pp. 289-556. In: S.M. Sano \& S.P. Almeida (eds.). Cerrado: ambiente e flora. Planaltina, Embrapa-CPAC.

Mendonça, R.C.; Felfili, J.M.; Walter, B.M.T.; Silva Júnior, M.C.; Rezende, A.V.; Filgueiras, T.S., Nogueira, P.E. \& Fagg, C.W. 2008. Flora vascular do Bioma Cerrado: checklist com 12.356 espécies. Pp. 423-1279. In: S.M. Sano, S.P. Almeida, J.F. Ribeiro (eds.). Cerrado: ecologia e flora. Brasília, Embrapa Cerrados.

Milliken, W. 1998. Structure and composition of one hectare of central Amazonian terra firme forest. Biotropica 30: 530-537.

Miranda, I.S. \& Absy, M.L. 1997. A flora fanerogâmica das savanas de Roraima. Pp. 445-462. In: R.I. Barbosa; E.J.G. Ferreira \& E.G. Castellõn (eds.). Homem, ambiente e ecologia no estado de Roraima. Manaus, INPA.

Miranda, I.S. \& Absy, M.L. 2000. Fisionomia das savanas de Roraima, Brasil. Acta Amazonica 30: 423-440.

Oliveira, A.A. de. 2000. Inventários quantitativos de árvores em matas de terra firme: histórico com enfoque na Amazônia brasileira. Acta Amazonica 30: 543-567.

Oliveira-Filho, A.T.; Jarenkow, J.A. \& Rodal, M.J.N. 2006. Floristic relationships of seasonally dry forests of Eastern South America based on tree species distribution patterns. Pp. 159-192. In: R.T Pennington; G.P. Lewis \& J.A. Ratter (eds.). Neotropical savannas and dry forests: plant diversity, biogeography, and conservation. Oxford, Taylor \& Francis CRC Press.

Pennington, R.T.; Lewis, G.P. \& Ratter, J.A. 2006. An overview of the plant diversity, biogeography and conservation of Neotropical savannas and seasonally dry forests. Pp. 1-29. In: R.T Pennington; G.P. Lewis \& J.A. Ratter (eds.). Neotropical savannas and dry forests: plant diversity, biogeography, and conservation. Oxford, Taylor \& Francis CRC Press.

Queiroz, L.P. 2004. Flora de Grão-Mogol, Minas Gerais: Leguminosae. Boletim de Botânica da Universidade de São Paulo 22: 213 265.

Queiroz, L.P. 2006. The Brazilian caatinga: phytogeographical patterns inferred from distribution data of the Leguminosae. Pp. 121-157. In: R.T Pennington; G.P. Lewis \& J.A. Ratter (eds.). Neotropical savannas and dry forests: plant diversity, biogeography, and conservation. Oxford, Taylor \& Francis CRC Press.

Radambrasil, 1975. Folha NA.20. Boa Vista e partes da folha NA.21. Tumucumaque, NB.20 e NB.21 (Geologia, geomorfologia, pedologia, vegetação e uso potencial da terra). Rio de Janeiro, Ministério da Minas e Energia.

Ratter, J.A; Bridgewater, S. \& Ribeiro, J.F. 2006. Biodiversity patterns of the woody vegetation of the Brazilian Cerrado. Pp. 31-66. In: R.T Pennington; G.P. Lewis \& J.A. Ratter (eds.). Neotropical savannas and dry forests: plant diversity, biogeography, and conservation. Oxford, Taylor \& Francis CRC Press.

Salomão, R. de P.; Silva, M.F.F. \& Rosa, N.A. 1988. Inventário ecológico em floresta pluvial tropical de terra firme, Serra Norte, Carajás, Pará. Boletim do Museu Paraense Emílio Goeldi, série Botânica 4: 1-46. 
Sette-Silva, E.L. 1997. A vegetação de Roraima. Pp. 401-415. In: R.I. Barbosa; E.J.G. Ferreira \& E.G. Castellõn (eds.). Homem, ambiente e ecologia no estado de Roraima. Manaus, INPA.

Silva, E.D. 2005. A subfamília Papilionoideae (Leguminosae Adans.) na Serra do Cabral, Minas Gerais. Dissertação de Mestrado, Universidade Estadual de Campinas.
Terborgh, J. \& Andresen, E. 1998. The composition of Amazonian forests: patterns at local and regional scales. Journal of Tropical Ecology 14: 645-664

Zappi, D.C.; Lucas, E.; Stannard, B.L.; Lughadha, E.N.; Pirani, J.R.; Queiroz, L.P.; Atkins, S.; Hind, D.J.N.; Giulietti, A.M.; Harley, R.M. \& Carvalho, A.M. 2003. Lista das plantas vasculares de Catolés, Chapada Diamantina, Bahia, Brasil. Boletim de Botânica da Universidade de São Paulo 21: 345-398. 